bies intabejondere aud Riedenburg zetgt. Wo bie Rorbjaumberjüngung aber allein zur 2lnwendung fommt, befürdfte idy für bie Wirtichaft eine (Sebunbentheit, bie id weber für erwünicht nod für nötig balte, wie Das bie [4hönen Erfolge in Seeftetten, Dietramzzell und Rangenbrand

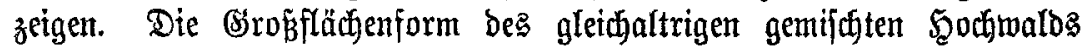

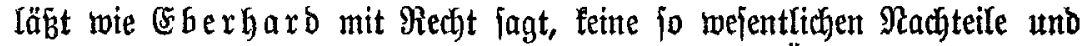

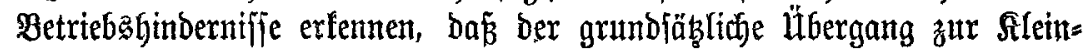
flächenform, wie iłn $\mathfrak{B a g n e r}$ forbert, begritmoet eridjene. $\mathfrak{B o}$ das aber

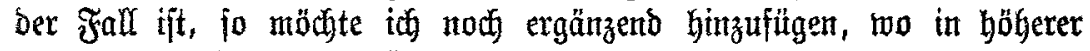

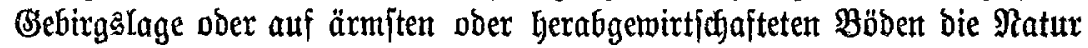
jelbft auf plenterartige Bemirtichaftung hinorängt, foll mant andererieit?

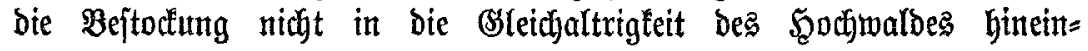
z̧wängen.

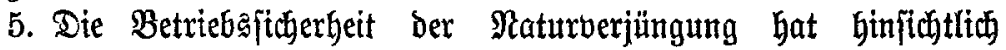

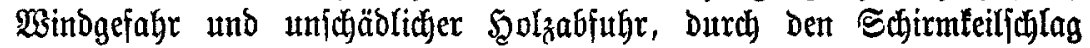

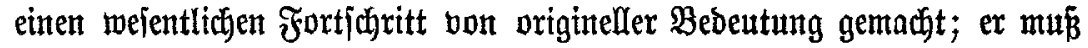
allerbings zur endgültigen $\mathfrak{B}$ eurteilung noch in reinen ober wentg ge= mif̧hten Fichtenbejtäntoen exprobt weroen. -

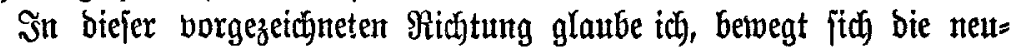
zeitliche Enttwifflung ber Naturberjüngung. Bon (F. L. Sartig ging

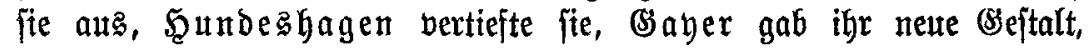
Wagner unb Eberhard zeigten nette Wege, bie uns wieder näber zum 马iele fübren twerden, wenn aud heute noch bie Semmniffe une oft zut erorütten orohen.

\title{
Die Strenfauna.
}

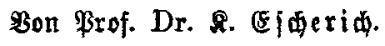

(Mit 1 MEb.)

Irs eime Der vornefmften 9 lufgaben Der Foritentomologie betradate

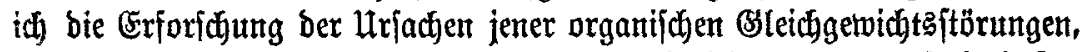
die zu Injeftentalamitäten fübren. Die Erforichung hat auj breitefter Bajiz zu geidehen, fie mus alle nur benffaren Faftorent berüdfitdigent unto mit möglidfft verfoujiedenen Methoden uno von möglichjt ver= \{ajiedenen Bsejichtapuntten aus in Ingriff genommen werden: genautelte Einzelerforfajung ber Srbäblinge und jebes iłrer \$arafiten, an möglidjt

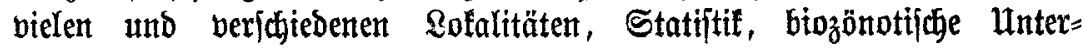
juffungen ujw. 
Bu Yeb̨teren gebört unter anberem ${ }^{1}$ ) bie (arforidung ber $8 \mathfrak{u}\{a$ mmen: jebung ber Fauna ber Streubede bezm. bes tierifhen $\mathfrak{s}$ halts ber Stren. Merfmürbigermeife ift biefez Thema bis jeb̧t nod faum

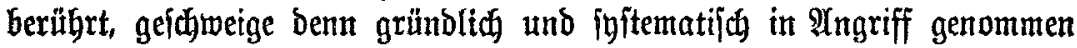
worden, obwohl boch bie Frage für bie \$raxis von höchitem Intereffe

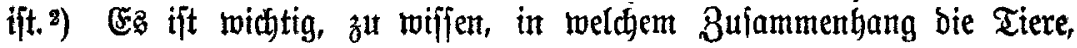
bie in ber Streu leben, mit ber Entftebung von Sialamitäten jtegen, b. h. ob fie nüblich (alfo gleidggewichtzerhaltend) oder füblorich (alfo gleich= gewichtaftiörend) wirfert ober böllig inbifferent find, und wie bern= entiprectend bom zoologificen Standpunt a

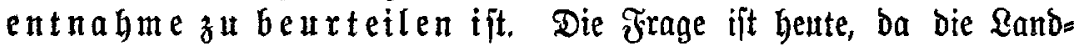

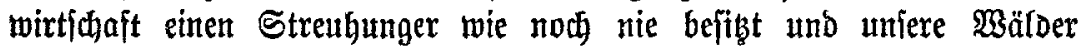
immer mehr Der Streubecte beraubt werben, bejonders aftuell.

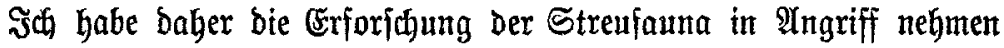

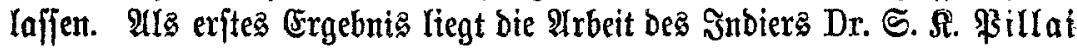
bor, ber mährend beş Siriegez mehrere sabre in ber zoplogifhen $\mathfrak{A} b=$

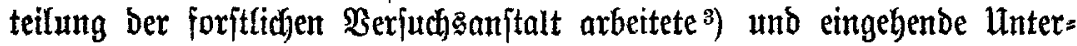
fuchungen über bie $\mathfrak{R}$ i f f er $\mathfrak{n}$ itre an anfellte.

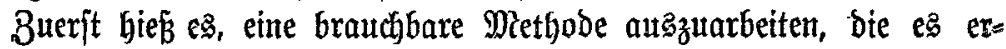
möglichte, aus großgen Streuquantitäten in lurzer Beit ben tierijojen

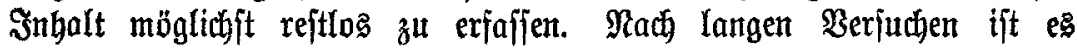
Billa $\mathfrak{i}$ gelungen, eine redbt brauthbare Methode ausfindig zu madhen. Şch will hier nicht auf bie (sinzelheiten eingeben (bieje find in Sriginal

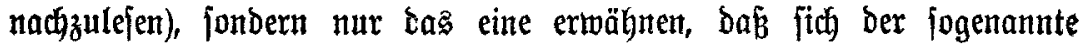

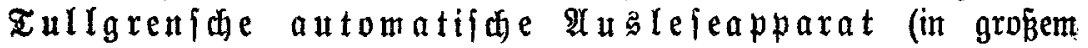

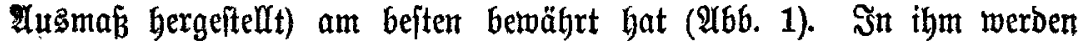
bie lebenben Tiere aus ber Streu burch rajde 2lustroctmung berjelben (mittels ciner 200 Яeronlampe) burd) eit Sieb in einen barunter be= findliden Tridbter getrieben und in einem Sammelglaz aufgefangen.

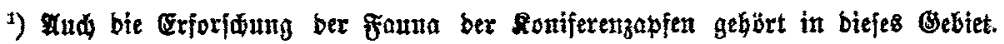

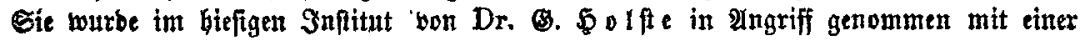
unterjudung über bie Bewobner ber Fidtenzapfer. Die 2rbeit ift im gleiden Feft bes 3eitfdrift fïr angewanbte Entomologie (BBb. VIII, 5eft 1) eridienen, wie bie bier bec iprodene Arbeit $\mathfrak{B}$ iff $a$ is. Dr. Solfte wirb über feine Ergebniffe biex felbft beriditen.

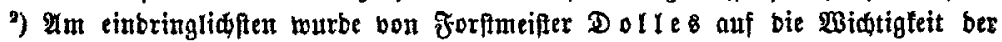
Streufauna für bie Erbaltung bes orgnniiden Bleidgetwidtbzuftanbeb bingemiejen (Jorft= unb naturmiffenjanftlide Beitjdrift 1897, S. 257-270).

•) S. R. \$ill $\mathfrak{a} \dot{\mathfrak{i}}$, Beiträge zur Renntni ber Fauna ber Walbftren, Riefern=

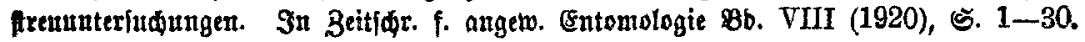
MRit 12 Mbb. unb 25 rabellen. 
Die Streu wurbe aut verichiedenen (Segenden ${ }^{1}$ ) und żt $^{\mathfrak{u}}$ ver=

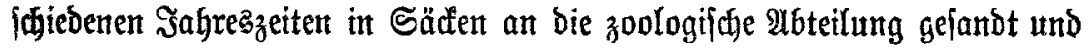
ztwar je 1 qm Streut in einem Sact. (Senaue 2 ngahen über bie \&age be

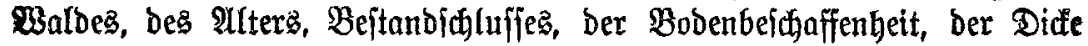
ber Streu[djidt und ber Be[tandteile, endlid ob berectit ober unberedit,

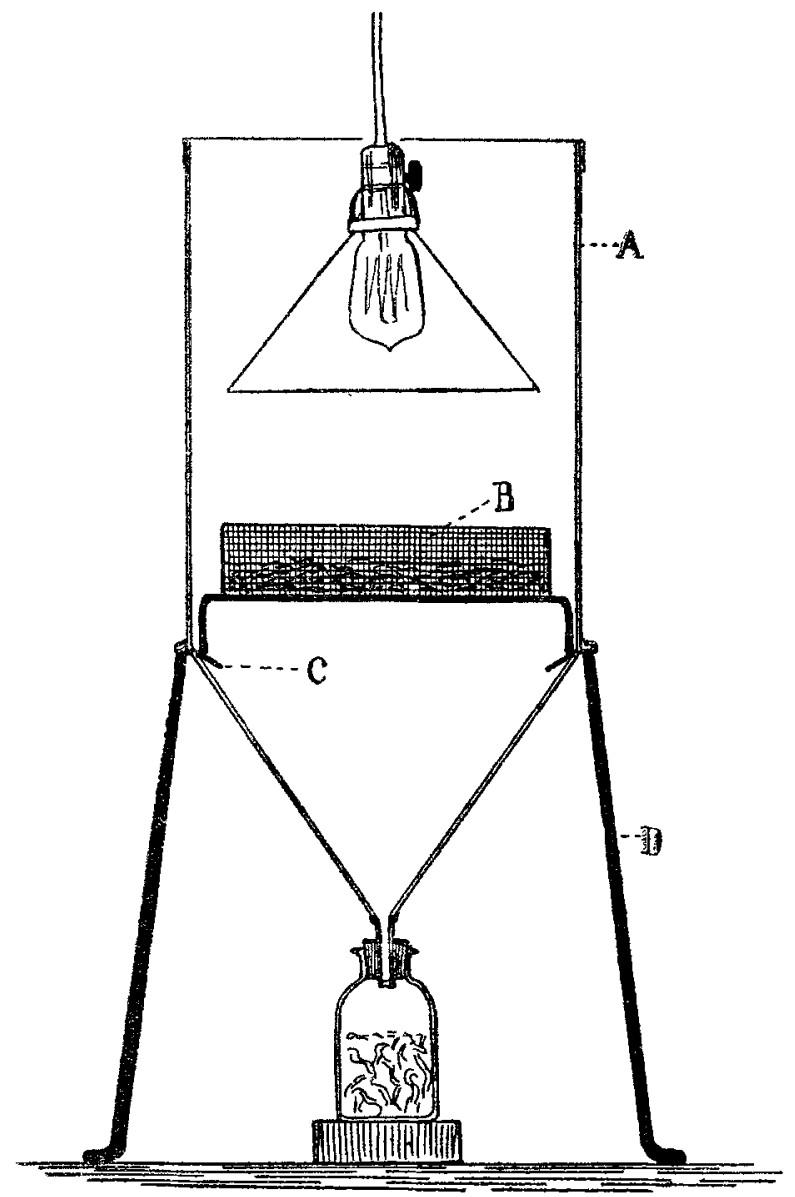

Mb5. 1.

waren jebem Sact beigegeben. Şm ganzen wurben tm Laufe eine \$abres 24 derartige Proben eingejandt und unterjuctit.

Die Rejultate ber fehr mühfamen und zeitraubenden Unterjuchungen finb in Form von Tabellen bargeitellt. $\mathfrak{A}$ us ibnen ergibt fitch zunädjit

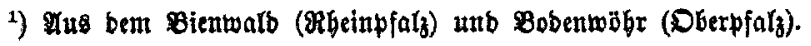




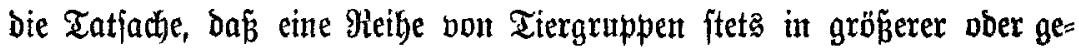
ringerer Menge in ber Streu vorbanden find, aljo einen darafteriftifdett Beftand ber Fitejernjtren barjtellen, währent anbere nur ba unb bort in einzelnen Sndibibuen vorbanden find und wohl mebr ober weniger $\mathfrak{z}^{\mathfrak{u}=}$ fällige Eriłfeinungen find.

3u Den exiteren, Den CGgaraftertieren Der Riefernjtreu, gehören: Dte Rematoden, Anneliben, کpinnen, Afterforpione, Milben, CGilopoben, Ipterggoten, Staphyliniden (Rarben und Jma= gines), die (Elavicornier (Rarben und Jimaginea), Elateriben (Rarven), Formiciden, Tenthreditiden (\$̧uppen), (Eecioomy= iden (Rarben), Tachinen (Puppen) und andere Dipteren (Rarben).

In teil's ungeheuren, oft nach vielen Taujenben pro Duabratmeter zählenden Mengen treten auf: Die Rlematoden, Milben, 2lpterygoten.

În grof̧en, meift naç f̧underten pro Duabratmeter zäblendent Mengen treten fajt ftets auf: Die Cectbomyiben=\&arben.

Die übrigen fanben fth meijt in geringerer $8 \mathfrak{Z} \mathfrak{h l}$ (ca. 5-100 pro Duabratmetex).

Unter ben $\mathfrak{M}$ i l ben waren folgende Familien vertreten: bie Parasitidae, Labidostomidae, Oribatidae und Trombididae.

Unter ben âpterygoten maden reitauz bie Mehrzahl bie Spritgifhtwänz (Eollembolen) aus, gegen bie bie anberen Famillen an 3ahl ganz zurühftreten.

Unter ben Elateriben= \&arben find vertreten bie ffattungen Dolopius, Athous, Elater und Corymbites.

Unter ben Formictben überwiegen bie Minrmiciben (Grattung Myrmica).

Bet Den Tenthrebiniben hanbelt eg fith authahmelos um Lophyrus: Eocons.

Untex Den Dipteren=Rarben befinden fith vertreten bie Dolichopodidae, Therevidae, Chironomidae, Leptis, Muscidae, Limnophilidae und Bibionidae.

Fragen wir mun, in welcher Beziehutng Die hier genannten હharatter=

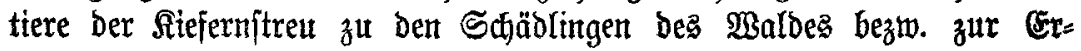

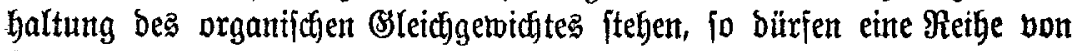
Titeren wobl bon vornberein ala "unbeteiligt" beztw. in bifferent aub geldyaltet werben: nämlich die anneliben, Apterygoten, ble Eeciónyiben und bie übrigen $D$ ipteren=Rarbent.

Đb bie Streu=:ematoben irgend welde Beziefungen zu bent

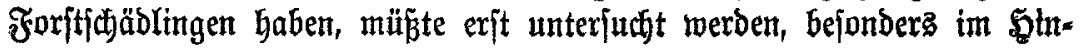




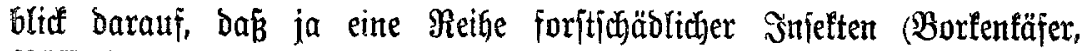
Rüffelfäfer) Ftellentweife unter ftarfen Remtatodeninfeftionen leiben.

Mas bie Milb e n betrifft, fo find unter ben feitgeitellten Forment meift joldie, bie fich von Pflanzenitoffen und zerfallenen organiffen Reften näbren. Sie find bager für umịere Frage jebenfalls nur von untergeoroneter $\mathfrak{B}$ edentung.

Die Ulferfforpione leben räuberifich, ftellen aber entiprechend ifrer Sleinheit mur fleineren Tieren nadh, in umferem Falle wobl in ber Shauptiadbe den Collembolen und Milben. Da bieie foritentomologifd belangloz zu jein icheinen, fo bürftent auth die alferjforptone zu ben forftentomologifich mehr oder meniger indifferenten Tieren gehören.

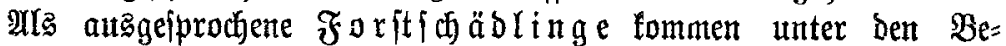
funben bor alfem bie Blattweipen (Lophyrus) in Setradyt, bie in jeber Streuprobe borbanden waren; ebenjo bie meift, went auth in ge= ringerer 8 ahl gefumbenen 5 w metterling

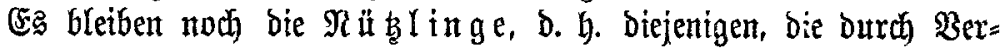

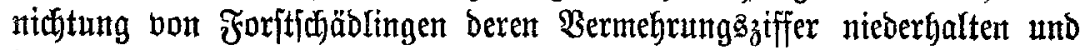

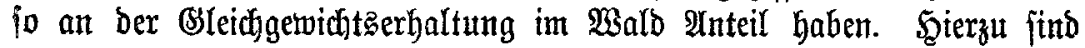
3t rechen: die Spinnen, Ehilopoden, Staphyliniben, Formictben und $\mathfrak{s}$ neumoniden.

Alle biefe finden fith faft in jeber Streuprobe in 2 nzahl und ipielen ztweifello: eine nidit unwejentliche $\Re$ iolle, bie allerbings in einzelnen exft aufgeflärt werden müste.

Welche Bebeutung fommt ben Elateriben=Laryen zu, bie eimen io regelmäßzigen uno hervorragenden Inteil an ber Streufautta Gaben? Dieje Frage bedarf ebenfalf noch eines eingebenden Studiums.

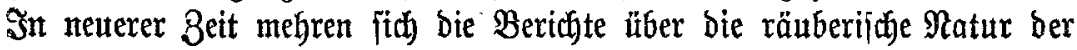

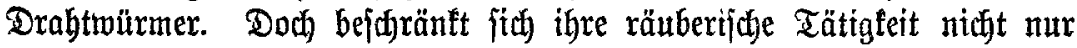

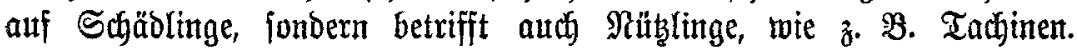

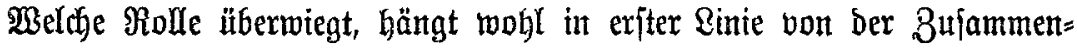

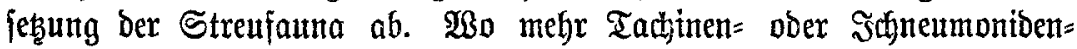

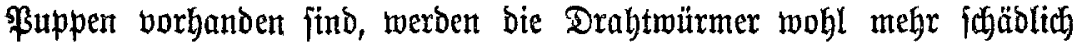
jein, wo mebr Shäbling spuppen vorbanden find, werben jie mehr nüblich

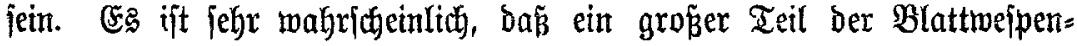
Cocons Durch Elateriden vernidytet wurben; wenigftens fpridgt bie Frorm ber in ben Eonconz gefreffenen Röber bafür. Daj bie Elateridenlarbent größere, fith bewegenbe Tiere anfallen, ift bet ifgrer ganzen Drganifation laum anzunebmen; fie werben mohl hauptfäd)lid an Rubejtabien fth beranmaden. -

Mas enblich die zablreidjen Clabicornier betrifit, bie fith faft 
in jeber Streuprobe vorfanbell, jo ift aud igre ßolle noch ungetlärt.

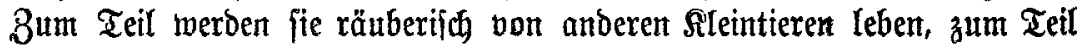
aber auth von pflanzlichem und fierijhem Detritus.

Die Unterjuchungen follen auch über bie Beränderungen bex Streu=

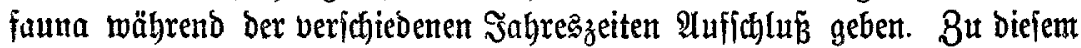
Zlwert werben zu berichiedenen Beiten Streuproben aub ber gleidjen

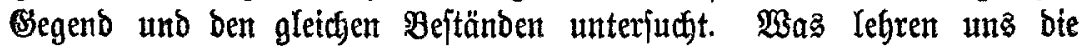
Ergebniffe?

Sie jind zmar verichieden, je nach Tiergruppen und Walbart (âtt= ober Stangenfol $z_{3}$, im allgemeinen jeboch connte folgendes feftgeftellt werben: Sm Sunt nebmen die Tiere gegenüber bem Bormonat an $8 \mathfrak{a b l}$ beträchtlich $\mathfrak{a b}$; im Julf fteigt ihre Babl wieber, um im September noch = mals zat fallen. Dttober zeigt Dann wieber eine Zunahme, wabrifjeinlich baburd hervorgerufen, bá bie Tiere zur überwinterung herunterfommen, Itm unter ber Strendecte in Den જooden zu gelangen. Sm Dezember wieberum finfen bie Surven, wohl beshalb, weil biele Tiere fith in ben mineralijhen Boden begebent haben.

Bergleichen wir ferner bie Strenfautua des âttholzez mit ber besి

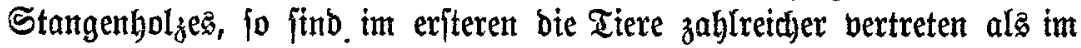

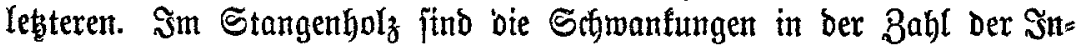

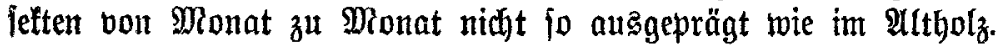

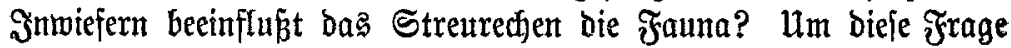
z̆ beantworten, wurbe Streu aus Bobenwöhr won beredtem und uns

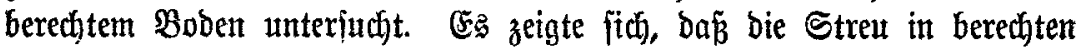
Wäldern im allgemeinen wentiger Tiere aufweilt, als in unberedten. Dan ftegt ferner, bas in erfterent eintge nüb̧liche Tiere weniger vertreten find als im unberechten $\mathfrak{B a l d}$. Dies trifft vor allem für bie Ebilo= poden, Spinnen, Staphylinen und Elateriben zu.

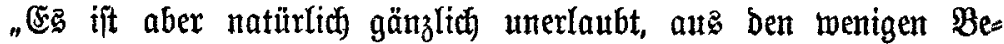

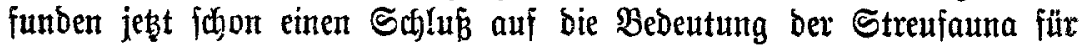
bie Erbaltung bes organifhen (Sleid)gewidtes zut ziehen, etwa in bem

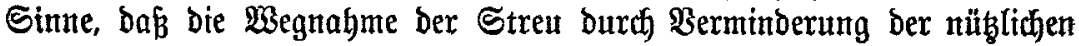
Tiere eine Bermebrung ber Sab̈blinge begünjtige. $3 \mathfrak{u}$ einem

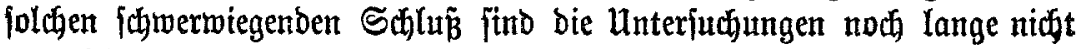

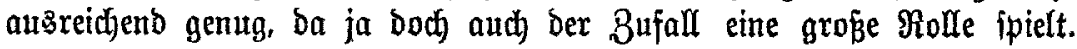
(Erit wenn bie Unterfucfungen in fo groper 8 ahl gemadt find, ba 8ufallsmoment als auggeidaltet betradtet werben tarn, werben berartige S๙hlüifie eine Berechtiguttg befommen“. -

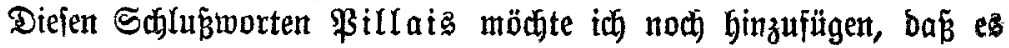
feine Qlbftht war, bas Thema nod weit eingebender zat bearbeiten the 


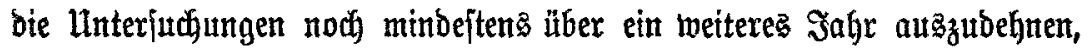
als er im \$erbjt 1918 plöblitid gezmungen war, Deutidjland z̆t verlaffen und feine ârbeit vorzeitig abzubrect)en. Wern aud infolgedeffen \$illais Arbeit unvollentet geblieben ift, fo jtellen feine llnterfuchungent boch eine

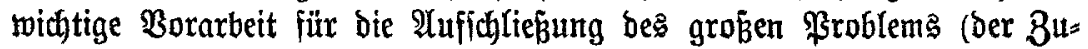
fammenfebzung ber Streufauma) bar, bie bann weiteren Unterjucfungen

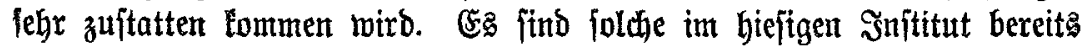
in $\mathfrak{A}$ (ngriff gentommen und ztwar über bie Fichten= und Budfenitreu, io

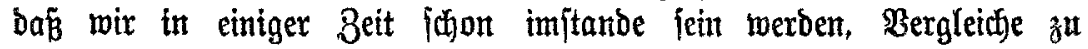
siefgen. -

\section{II. dittexurifde exinte.}

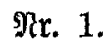

9litteilumgen ber Sdjweizerifjen Centralanjtalt fït bas farit:

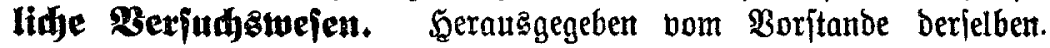
Dr. Arnold (Engler, Frofeffor an ber (sibg. Techn. Sgochidule in Büric. XI. Banto, 3. Geft. Mii 12 Bildern. Bürid, Sommifftong= verlag von Beer \& Co., 1921.

Das Şeft enthält 2 Yrbeiten:

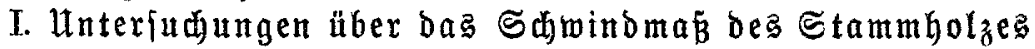
bei $\mathfrak{B i n t e r}=\mathfrak{u n d}$ Sommerfällung. Bon Dr. Bhilipp Flury, Olojunft ber Eitog. forftl. Berfuchanitalt.

II. Über morphologifale und biologifue Eigenichaften ber Stiel= und Traubeneidhe unb igre Erziebung im Forit:

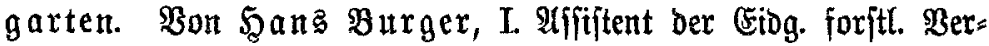
juchsanitalt.

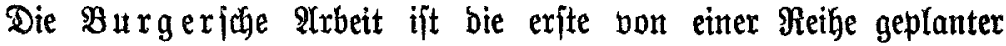

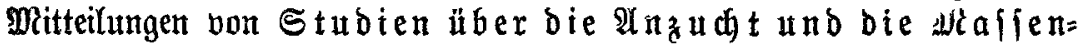

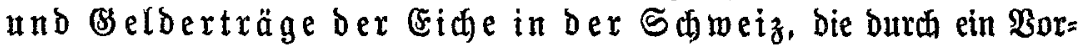
mort bon $\mathfrak{A}$. F $\mathfrak{n} g \mathfrak{l} \mathfrak{e} \mathfrak{x}$ eitngeleitet find.

$\mathfrak{W a g}$ zunächjt Die Unterjudungen von $\mathfrak{F} \mathfrak{l}$ ury anlangt, fo lautete

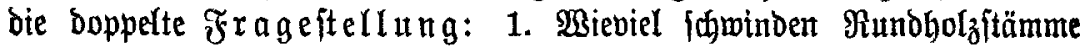

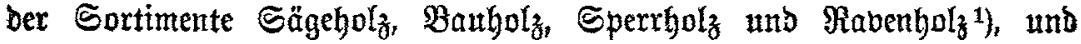
Etangen vom frifdgefällten bis zum walotrodenen Zujtand und żwar bei Winter = und bei Sommerfällung, twenn bie Stämme im Freien ge=

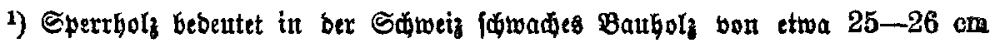

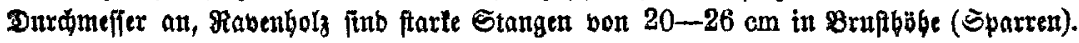

